\title{
Краевая задача для уравнения смешанного типа с сингулярным коэффициентом
}

\section{М. Х. Рузиев ${ }^{1}$, Ф. С. Актамов ${ }^{2}$}

1 Институт Математики АН РУз, 100174, г. Ташкент, ул. Университетская, 4б

2 Чирчикский государственный педагогический институт, 111700, г. Чирчик, пр-т. Амира Темура, 104, Узбекистан

E-mail: mruziev@mail.ru

В работе изучается краевая задача для уравнения смешанного типа с сингулярным коэффициентом в области, эллиптической частью которой является первая четверть плоскости, а гиперболической частью - характеристический треугольник. Методами интегральных уравнений и принципа экстремума доказывается однозначная разрешимость рассматриваемой задачи.

Ключевые слова: принцип экстремума, единственность решения, существование решения, сингулярный коэффициент, индекс уравнения

DOI: $10.26117 / 2079-6641-2021-35-2-27-39$

Поступила в редакцию: 24.04.2021

В окончательном варианте: 04.06.2021

Для цитирования. Рузиев М. Х., Актамов Ф. С. Краевая задача для уравнения смешанного типа с сингулярным коэффициентом // Вестник КРАУНЦ. Физ.-мат. науки. 2021. Т. 35. № 2. C. 27-39. DOI: 10.26117/2079-6641-2021-35-2-27-39

Контент публикуется на условиях лицензии Creative Commons Attribution 4.0 International (https://creativecommons.org/licenses/by/4.0/deed.ru)

(C) Рузиев М.X., Актамов Ф.С., 2021

\section{Введение и постановка задачи}

Рассмотрим уравнение

$$
\operatorname{signy}|y|^{m} u_{x x}+u_{y y}+\frac{\beta_{0}}{y} u_{y}=0
$$

в области $D=D^{+} \cup D^{-} \cup I$ комплексной плоскости $z=x+i y$, где $D^{+}-$первый квадрант плоскости, $D^{-}-$конечная область четвертого квадранта плоскости, ограниченная характеристиками $O C$ и $B C$ уравнения (1), выходящими из точек $O(0,0), B(1,0)$, и отрезком $O B$ прямой $y=0, I=\{(x, y): 0<x<1, y=0\}$. В (1) $m, \beta_{0}-$ некоторые действительные числа, удовлетворяющие условиям $m>0,-\frac{m}{2}<\beta_{0}<1$.

Введем обозначения: $I_{0}=\{(x, y): 0<y<\infty, x=0\}, I_{1}=\{(x, y): 1<x<\infty, y=0\}, C_{0}$ и $C_{1}-$ соответственно точки пересечения характеристик $O C$ и $B C$ с характеристикой, исходящей из точки $E(c, 0)$, где $c \in I-$ произвольное фиксированное число.

Финансирование. Исследование выполнялось без финансовой поддержки фондов. 
Пусть $p(x) \in C^{1}[0, c]-$ диффеоморфизм из множества точек отрезка $[0, c]$ в множество точек отрезка $[c, 1]$, причем $p^{\prime}(x)<0, p(0)=1, p(c)=c$. В качестве примера такой функции приведем линейную функцию $p(x)=1-k x$, где $k=\frac{1-c}{c}$.

Задача В. Найти в области $D$ функцию $u(x, y)$ со свойствами:

1) $u(x, y) \in C(\bar{D})$ где $\bar{D}=\bar{D}^{-} \cup D^{+} \cup \bar{I}_{0} \cup \overline{I_{1}}$;

2) $u(x, y) \in C^{2}\left(D^{+}\right)$и удовлетворяет уравнению (1) в этой области;

3) $u(x, y)$ является обобщенным решением класса $R_{1}$ [1] в области $D^{-}$;

4) выполняются равенства

$$
\lim _{R \rightarrow \infty} u(x, y)=0, R^{2}=x^{2}+\frac{4}{(m+2)^{2}} y^{m+2}, x \geq 0, y \geq 0
$$

5) $u(x, y)$ удовлетворяет краевым условиям

$$
\begin{gathered}
u(0, y)=\varphi(y), y \geq 0, \\
u(x, 0)=\tau_{1}(x), x \in \bar{I}_{1}, \\
\left.u(x, y)\right|_{E C_{0}}=\psi(x), \frac{c}{2} \leq x \leq c, \\
u(p(x), 0)=\mu u(x, 0)+f(x), 0 \leq x \leq c,
\end{gathered}
$$

и условию сопряжения

$$
\lim _{y \rightarrow+0} y^{\beta_{0}} \frac{\partial u}{\partial y}=\lim _{y \rightarrow-0}(-y)^{\beta_{0}} \frac{\partial u}{\partial y}, \quad x \in I \backslash\{c\},
$$

причем эти пределы при $x=0, x=1, x=c$ могут иметь особенности порядка ниже $1-2 \beta$, где $\beta=\frac{m+2 \beta_{0}}{2(m+2)}, f(x) \in C[0, c] \cap C^{1, \delta_{1}}(0, c), f(0)=0, f(c)=0, \psi(x) \in$ $C\left[\frac{c}{2}, c\right] \cap C^{1, \delta_{2}}\left(\frac{c}{2}, c\right), \quad \psi(c)=0, \tau_{1}(x) \in C\left(\bar{I}_{1}\right)$, причем функция $\tau_{1}(x)$ в окрестности точки $x=1$ представима в виде $\tau_{1}(x)=(1-x) \tau_{1}(x), \tilde{\tau}_{1}(x) \in C\left(\bar{I}_{1}\right)$ и при достаточно больших $x$ удовлетворяет неравенству $\left|\tau_{1}(x)\right| \leq \frac{M}{x^{\varepsilon}}, \varepsilon, M$ - положительные константы, $\tau_{1}(x)$ удовлетворяет условию Гельдера на любом отрезке $[1, N], N>1, \varphi(y) \in C\left(\bar{I}_{0}\right)$, $y^{\frac{3 m+2 \beta_{0}}{4}} \varphi(y) \in L(0, \infty), \varphi(y)$ удовлетворяет условию Гельдера на любом отрезке $[0, N]$, $N>0, \varphi(\infty)=0, \varphi(0)=0$.

Краевая задача со смещением для уравнения смешанного типа в неограниченной области, эллиптическая часть которой - верхняя полуплоскость, исследована в работе [2]. Краевая задача для уравнения (1) в смешанной области, эллиптическая часть которой - полуполоса, решена в работе [3]. Отметим, что в задаче Геллерстедта [4] значение искомой функции в гиперболической части смешанной области $D$ задается на характеристиках $E C_{0}$ и $E C_{1}$ :

$$
\left.u\right|_{E C_{0}}=\psi_{1}(x),\left.u\right|_{E C_{1}}=\psi_{2}(x) .
$$

В настоящей работе исследуется задача, где внутренняя характеристика $E C_{1}$ освобождена от краевого условия, и это недостающее условие Геллерстедта заменено внутренним краевым условием локального смещения на отрезке $A B$ линии вырождения $y=0$. Отметим, что условие (6) является внутренне краевым условием локального смещения на отрезке линии параболического вырождения [5], [6], [7]. 


\section{Единственность решения задачи}

Теорема 1. Пусть выполнень условия $\varphi(y) \equiv 0, \psi(x) \equiv 0, f(x) \equiv 0, \tau_{1}(x) \equiv 0$, $0<\mu<1$. Тогда задача $B$ имеет лишь тривиальное решение.

Доказательство. Известно, что решение видоизмененной задачи Коши $u(x, 0)=$ $\tau(x), x \in \bar{I}, \lim _{y \rightarrow-0}(-y)^{\beta_{0}} u_{y}=v(x), x \in I$, имеет вид

$$
\begin{aligned}
& u(x, y)=\gamma_{1} \int_{0}^{1} \tau\left(x+\frac{2}{m+2}(-y)^{\frac{m+2}{2}}(2 t-1)\right) t^{\beta-1}(1-t)^{\beta-1} d t \\
& -\gamma_{2}(-y)^{1-\beta_{0}} \int_{0}^{1} v\left(x+\frac{2}{m+2}(-y)^{\frac{m+2}{2}}(2 t-1)\right) t^{-\beta}(1-t)^{-\beta} d t
\end{aligned}
$$

где $\gamma_{1}=\frac{\Gamma(2 \beta)}{\Gamma^{2}(\beta)}, \gamma_{2}=\frac{2}{(m+2)} \frac{\Gamma(1-2 \beta)}{\Gamma^{2}(1-\beta)}, \Gamma(z)$-гамма функция [1]. В силу формулы (8), из краевого условия (5) после несложных вычислений получим

$$
v(X)=\gamma D_{X, c}^{1-2 \beta} \tau(X)+\Psi(X), X \in(0, c),
$$

$\Psi(X)=-\frac{(c-X)^{\beta} D_{X, c}^{1-\beta} \psi\left(\frac{X+c}{2}\right)}{\gamma_{2}\left(\frac{m+2}{4}\right)^{1-2 \beta} \Gamma(1-\beta)}, \quad \gamma=\frac{2 \Gamma(1-\beta) \Gamma(2 \beta)}{\Gamma(\beta) \Gamma(1-2 \beta)}\left(\frac{m+2}{4}\right)^{2 \beta}, X=2 x-c, D_{X, c}^{l} \quad-\quad$ оператор дробного дифференцирования в смысле Римана-Лиувилля [1].

Равенство (9) является первым функциональным соотношением между неизвестными функциями $\tau(x)$ и $v(x)$, принесенным на интервал $(0, c)$ оси $y=0$ из гиперболической части $D^{-}$смешанной области $D$. Теперь докажем, что если $\varphi(y) \equiv 0$, $\psi(x) \equiv 0, f(x) \equiv 0, \tau_{1}(x) \equiv 0,0<\mu<1$, то решение задачи $B$ в области $D^{+} \cup I_{0} \cup \bar{I} \cup I_{1}$ в силу (2) тождественно равно нулю. Пусть $\left(x_{0}, y_{0}\right)$ - точка положительного максимума функции $u(x, y)$ в области $\bar{D}_{R}^{+}$.

Пусть $D_{R}^{+}-$конечная область, отсекаемая от области $D^{+}$дугой $A_{R} B_{R}$ нормальной кривой $x^{2}+\frac{4}{(m+2)^{2}} y^{m+2}=R^{2}, 0 \leq x \leq R, 0 \leq y \leq\left(\frac{m+2}{2} R\right)^{2 /(m+2)}$, где $A_{R}$ и $B_{R}$ точки с координатами $(0, R)$ и $(R, 0)$ соответственно. В силу (2) для любого $\varepsilon>0$ существует такое $R_{0}=R_{0}(\varepsilon)$, что при $R>R_{0}(\varepsilon)$ выполняется неравенство

$$
|u(x, y)|<\varepsilon,(x, y) \in A_{R} B_{R} .
$$

В силу обозначения $u(x, 0)=\tau(x), \quad x \in \bar{I}$ условие (6) перепишем в виде

$$
\tau(p(x))=\mu \tau(x)+f(x), x \in[0, c] .
$$

Отсюда при $x=c$ (где $f(x) \equiv 0)$ имеем $\tau(p(c))=\mu \tau(c)$. Тогда в силу равенства $p(c)=c$ следует, что $\tau(c)(1-\mu)=0$, т.е. $\tau(c)=0$. По принципу Хопфа [8], функция $u(x, y)$ своего положительного максимума и отрицательного минимума во внутренних точках области $\bar{D}_{R}^{+}$не достигает. В силу $0<\mu<1$ из (11) (где $\left.f(x) \equiv 0\right)$ следует, что их также нет и в интервале $(c, 1)$ оси $y=0$.

Пусть $\left(x_{0}, 0\right) \quad\left(\right.$ ге $\left.x_{0} \in(-1, c)\right)$ - точка положительного максимума (отрицательного минимума) функции $u(x, 0)=\tau(x)$. Тогда в этой точке в случае положительного максимума(отрицательного минимума) [5]

$$
v\left(x_{0}\right)<0\left(v\left(x_{0}\right)>0\right) .
$$


Хорошо известно, что в точке положительного максимума (отрицательного минимума) функции $\tau(x)$ для операторов дробного дифференцирования имеет место неравенство $\left.D_{x, c}^{1-2 \beta} \tau(x)\right|_{x=x_{0}} \tau(x)>0\left(\left.D_{x, c}^{1-2 \beta} \tau(x)\right|_{x=x_{0}} \tau(x)<0\right)$. Тогда в силу (9) (где $\Psi(x) \equiv 0)$

$$
v\left(x_{0}\right)=\left.\gamma D_{x, c}^{1-2 \beta} \tau(x)\right|_{x=x_{0}}>0\left(v\left(x_{0}\right)=\left.\gamma D_{x, c}^{1-2 \beta} \tau(x)\right|_{x=x_{0}}<0\right) .
$$

Неравенства (12) и (13) противоречат условию сопряжения (7), отсюда следует, что $x_{0} \notin(0, c)$. Следовательно, точки положительного максимума (отрицательного минимума) функции $u(x, y)$ нет на интервале $A B$. Пусть $R>R_{0}$. Из принципа Хопфа и предыдущих рассуждений получаем, что если $\left(x_{0}, y_{0}\right) \in A_{R} B_{R}$, то в силу (10) $\left|u\left(x_{0}, y_{0}\right)\right|<\varepsilon$. Следовательно, $|u(x, y)|<\varepsilon$ для любых $(x, y) \in \bar{D}_{R}^{+}$. Отсюда в силу произвольности $\varepsilon$ при $R \rightarrow+\infty$ заключаем, что $u(x, y) \equiv 0$ в области $D^{+} \cup I_{0} \cup \bar{I} \cup I_{1}$. Тогда

$$
\lim _{y \rightarrow+0} u(x, y)=0, x \in \bar{I} ; \lim _{y \rightarrow+0} y^{\beta_{0}} u_{y}=0, x \in I
$$

С учетом (14), в силу непрерывности решения в области $\bar{D}_{R}^{+}$и условия сопряжения (7), восстанавливая искомую функцию $u(x, y)$ в области $D^{-}$как решение видоизмененной задачи Коши с однородными данными, получим $u(x, y) \equiv 0$ в области $\bar{D}^{-}$.

\section{Существование решения задачи}

Теорема 2. Пусть выполнены условия $\mu k^{\frac{1}{2}-3 \alpha} \sin (\alpha \pi)<1$, где $\alpha=(1-2 \beta) / 4$, $\beta_{0}>\frac{1-m}{3}, p(x)=1-k x$. Тогда решение задачи $B$ существует.

Доказательство. Решение задачи Дирихле в области $D^{+}$, удовлетворяющее условиям (2)-(4) и условию $u(x, 0)=\tau(x), x \in \bar{I}$, представимо в виде

$$
\begin{aligned}
& u(x, y)=k_{2} y^{1-\beta_{0}} \int_{0}^{1} \tau(t) \\
& \times\left(\left((t-x)^{2}+\frac{4}{(m+2)^{2}} y^{m+2}\right)^{\beta-1}-\left((t+x)^{2}+\frac{4}{(m+2)^{2}} y^{m+2}\right)^{\beta-1}\right) d t \\
& +k_{2} y^{1-\beta_{0}} \int_{1}^{\infty} \tau_{1}(t) \\
& \times\left(\left((t-x)^{2}+\frac{4}{(m+2)^{2}} y^{m+2}\right)^{\beta-1}-\left((t+x)^{2}+\frac{4}{(m+2)^{2}} y^{m+2}\right)^{\beta-1}\right) d t \\
& +\frac{2}{m+2} y^{\frac{1-\beta_{0}}{2}} \int_{0}^{\infty} t^{\frac{2 m+1+\beta_{0}}{2}} \varphi(t) d t \\
& \times \int_{0}^{\infty} s e^{-s x} J_{\frac{1-2 \beta}{2}}\left(\frac{2 s t^{\frac{m+2}{2}}}{m+2}\right) J_{\frac{1-2 \beta}{2}}\left(\frac{2 s y^{\frac{m+2}{2}}}{m+2}\right) d s,
\end{aligned}
$$

где $k_{2}=\frac{1}{4 \pi}\left(\frac{4}{m+2}\right)^{2-2 \beta} \frac{\Gamma^{2}(1-\beta)\left(1-\beta_{0}\right)}{\Gamma(2-2 \beta)}, \beta=\frac{2 \beta_{0}+m}{2(m+2)}, J_{v}(z)$ - функция Бесселя первого рода. 
Дифференцируя равенство (15) по $y$, получим

$$
\begin{aligned}
& \frac{\partial u}{\partial y}=k_{2} \int_{0}^{1} \tau(t) \frac{\partial}{\partial y} y^{1-\beta_{0}} \\
& \times\left(\left((t-x)^{2}+\frac{4}{(m+2)^{2}} y^{m+2}\right)^{\beta-1}-\left((t+x)^{2}+\frac{4}{(m+2)^{2}} y^{m+2}\right)^{\beta-1}\right) d t \\
& +\frac{\partial}{\partial y} F_{1}(x, y)+\frac{\partial}{\partial y} F_{2}(x, y)
\end{aligned}
$$

где

$$
F_{1}(x, y)=k_{2} \int_{1}^{\infty} \tau_{1}(t) y^{1-\beta_{0}}
$$

$\times$

$$
\begin{gathered}
\left(\left((t-x)^{2}+\frac{4}{(m+2)^{2}} y^{m+2}\right)^{\beta-1}-\left((t+x)^{2}+\frac{4}{(m+2)^{2}} y^{m+2}\right)^{\beta-1}\right) d t, \\
F_{2}(x, y)=\frac{2}{m+2} y^{\frac{1-\beta_{0}}{2}} \int_{0}^{\infty} t^{\frac{2 m+1+\beta_{0}}{2}} \varphi(t) d t \int_{0}^{\infty} s e^{-s x} J_{\frac{1-2 \beta}{2}}\left(\frac{2 s t^{\frac{m+2}{2}}}{m+2}\right) J_{\frac{1-2 \beta}{2}}\left(\frac{2 s y^{\frac{m+2}{2}}}{m+2}\right) d s .
\end{gathered}
$$

В силу равенства

$$
\begin{gathered}
\frac{\partial}{\partial y} y^{1-\beta_{0}}\left(\left[(x-t)^{2}+\frac{4}{(m+2)^{2}} y^{m+2}\right]^{\beta-1}-\left[(x+t)^{2}+\frac{4}{(m+2)^{2}} y^{m+2}\right]^{\beta-1}\right)= \\
=\frac{m+2}{2} y^{-\beta_{0}} \frac{\partial}{\partial t} \times \\
\times\left((x-t)\left[(x-t)^{2}+\frac{4}{(m+2)^{2}} y^{m+2}\right]^{\beta-1}+(x+t)\left[(x+t)^{2}+\frac{4}{(m+2)^{2}} y^{m+2}\right]^{\beta-1}\right),
\end{gathered}
$$

из (16) имеем

$$
\begin{aligned}
& \frac{\partial u}{\partial y}=k_{2} \frac{m+2}{2} y^{-\beta_{0}} \int_{0}^{1} \tau(t) \\
& \times \frac{\partial}{\partial t}\left((x-t)\left[(x-t)^{2}+\frac{4}{(m+2)^{2}} y^{m+2}\right]^{\beta-1}+(x+t)\left[(x+t)^{2}+\frac{4}{(m+2)^{2}} y^{m+2}\right]^{\beta-1}\right) d t \\
& +\frac{\partial}{\partial y} F_{1}(x, y)+\frac{\partial}{\partial y} F_{2}(x, y) .
\end{aligned}
$$

В интеграле правой части равенства (17) выполнив операцию интегрирования по частям, с учетом $\tau(0)=0, \tau(1)=0$, после несложных вычислений имеем

$$
\begin{aligned}
& \frac{\partial u}{\partial y}=-k_{2} \frac{m+2}{2} y^{-\beta_{0}} \int_{0}^{1} \tau^{\prime}(t) \\
& \times\left((x-t)\left[(x-t)^{2}+\frac{4}{(m+2)^{2}} y^{m+2}\right]^{\beta-1}+(x+t)\left[(x+t)^{2}+\frac{4}{(m+2)^{2}} y^{m+2}\right]^{\beta-1}\right) d t \\
& +\frac{\partial}{\partial y} F_{1}(x, y)+\frac{\partial}{\partial y} F_{2}(x, y) .
\end{aligned}
$$


Умножая обе части равенства (18) на $y^{\beta_{0}}$, затем переходя к пределу при $y \rightarrow+0$, получим

$$
v(x)=-k_{2} \frac{m+2}{2} \int_{0}^{1} \tau^{\prime}(t)\left[(x-t)|x-t|^{2 \beta-2}+(t+x)^{2 \beta-1}\right] d t+\Phi_{0}(x), x \in(0,1),
$$

где

$$
\begin{aligned}
\Phi_{0}(x)= & \lim _{y \rightarrow+0} y^{\beta_{0}} \frac{\partial}{\partial y}\left(F_{1}(x, y)+F_{2}(x, y)\right)=k_{2}\left(1-\beta_{0}\right) \int_{0}^{\infty} \tau_{1}(t)\left[(t-x)^{2 \beta-2}-(t+x)^{2 \beta-2}\right] d t \\
& +\frac{2}{(m+2)^{\frac{1-2 \beta}{2}} \Gamma\left(\frac{1}{2}-\beta\right)} \int_{0}^{\infty} \varphi(t) t^{\frac{2 m+1+\beta_{0}}{2}} d t \int_{0}^{\infty} s^{\frac{3-2 \beta}{2}} e^{-s x} J_{\frac{1-2 \beta}{2}}\left(\frac{2 s^{\frac{m+2}{2}}}{m+2}\right) d s .
\end{aligned}
$$

Равенство (19) есть функциональное соотношение между неизвестными функциями $\tau(x)$ и $v(x)$, принесенное на $I$ из эллиптической части $D^{+}$смешанной области $D$. Заметим, что соотношение (19) справедливо для всего промежутка $I$. Далее, промежуток интегрирования $(0,1)$ разбивая на промежутки $(0, c)$ и $(c, 1)$, а затем в интегралах с пределом $(c, 1)$ сделав замену переменного интегрирования $t=p(s)=$ $1-k s$ и учитывая равенство (11), соотношение (19) приведем к виду

$$
\begin{aligned}
& v(x)=-k_{2} \frac{m+2}{2}\left(\int_{0}^{x} \tau^{\prime}(t)(x-t)^{2 \beta-1} d t-\int_{x}^{c} \tau^{\prime}(t)(t-x)^{2 \beta-1} d t\right) \\
& -k_{2} \frac{m+2}{2}\left(\int_{0}^{c} \tau^{\prime}(t)(t+x)^{2 \beta-1} d t+\mu \int_{0}^{c} \tau^{\prime}(s)\left[(p(s)-x)^{2 \beta-1}-(p(s)+x)^{2 \beta-1}\right] d s\right) \\
& +\Phi_{1}(x), x \in(0, c),
\end{aligned}
$$

где

$$
\Phi_{1}(x)=-k_{2} \frac{m+2}{2} \int_{0}^{c} f^{\prime}(s)\left[(p(s)-x)^{2 \beta-1}-(p(s)+x)^{2 \beta-1}\right] d s+\Phi_{0}(x)
$$

В силу (7), исключая функцию $v(x)$ из (9) и (20), получим

$$
\begin{aligned}
& \gamma D_{x, c}^{1-2 \beta} \tau(x)+\Psi(x)=-k_{2} \frac{m+2}{2}\left(\int_{0}^{x} \tau^{\prime}(t)(x-t)^{2 \beta-1} d t-\int_{x}^{c} \tau^{\prime}(t)(t-x)^{2 \beta-1} d t\right) \\
& -k_{2} \frac{m+2}{2}\left(\int_{0}^{c} \tau^{\prime}(t)(t+x)^{2 \beta-1} d t+\mu \int_{0}^{c} \tau^{\prime}(s)\left[(p(s)-x)^{2 \beta-1}-(p(s)+x)^{2 \beta-1}\right] d s\right) \\
& +\Phi_{1}(x), x \in(0, c) .
\end{aligned}
$$

Равенство (21) перепишем в виде

$$
\begin{gathered}
-\frac{2 \gamma}{(m+2) k_{2}} D_{x, c}^{1-2 \beta} \tau(x)+\Phi_{2}(x)=\int_{0}^{x} \tau^{\prime}(t)(x-t)^{2 \beta-1} d t-\int_{x}^{c} \tau^{\prime}(t)(t-x)^{2 \beta-1} d t+ \\
+\int_{0}^{c} \tau^{\prime}(t)(t+x)^{2 \beta-1} d t+\mu \int_{0}^{c} \tau^{\prime}(s)\left[(p(s)-x)^{2 \beta-1}-(p(s)+x)^{2 \beta-1}\right] d s, x \in(0, c),
\end{gathered}
$$


где $\Phi_{2}(x)=-\frac{2}{(m+2) k_{2}}\left(\Psi(x)-\Phi_{1}(x)\right)$. Применив оператор $\Gamma(1-2 \beta) D_{x, c}^{2 \beta-1} \quad$ к обеим частям равенства $(22)$ и учитывая, что $D_{x, c}^{2 \beta-1} D_{x, c}^{1-2 \beta} \tau(x)=\tau(x)$, имеем

$$
\begin{aligned}
& -\frac{2 \gamma}{(m+2) k_{2}} \Gamma(1-2 \beta) \tau(x)+\Gamma(1-2 \beta) D_{x, c}^{2 \beta-1} \Phi_{2}(x)=\Gamma(1-2 \beta) D_{x, c}^{2 \beta-1} \\
& \times\left(\int_{0}^{x} \tau^{\prime}(t)(x-t)^{2 \beta-1} d t-\int_{x}^{c} \tau^{\prime}(t)(t-x)^{2 \beta-1} d t\right)+\Gamma(1-2 \beta) D_{x, c}^{2 \beta-1} \\
& \times\left(\int_{0}^{c} \tau^{\prime}(t)(t+x)^{2 \beta-1} d t+\mu \int_{0}^{c} \tau^{\prime}(s)\left[(p(s)-x)^{2 \beta-1}-(p(s)+x)^{2 \beta-1}\right] d s\right) \\
& , x \in(0, c) .
\end{aligned}
$$

Нетрудно убедиться в том, что

$$
\begin{gathered}
\Gamma(1-2 \beta) D_{x, c}^{2 \beta-1} \int_{0}^{x} \tau^{\prime}(t)(x-t)^{2 \beta-1} d t=-\Gamma(2 \beta) \Gamma(1-2 \beta) \cos (2 \pi \beta) \tau(x) \\
-\int_{0}^{c}\left(\frac{c-x}{c-t}\right)^{1-2 \beta} \frac{\tau(t) d t}{t-x}, \\
\Gamma(1-2 \beta) D_{x, c}^{2 \beta-1} \int_{x}^{c} \tau^{\prime}(t)(t-x)^{2 \beta-1} d t=-\Gamma(2 \beta) \Gamma(1-2 \beta) \tau(x), \\
\Gamma(1-2 \beta) D_{x, c}^{2 \beta-1} \int_{0}^{c} \tau^{\prime}(t)(t+x)^{2 \beta-1} d t=\int_{0}^{c}\left(\frac{c-x}{c+t}\right)^{1-2 \beta} \frac{\tau(t) d t}{t+x}, \\
\Gamma(1-2 \beta) \mu D_{x, c}^{2 \beta-1} \int_{0}^{c} \tau^{\prime}(s)(p(s)-x)^{2 \beta-1} d s=\mu \int_{0}^{c}\left(\frac{c-x}{p(s)-c}\right)^{1-2 \beta} \frac{\tau(s) p^{\prime}(s) d s}{p(s)-x}, \\
\mu \Gamma(1-2 \beta) \mu D_{x, c}^{2 \beta-1} \int_{0}^{c} \tau^{\prime}(s)(p(s)+x)^{2 \beta-1} d s=\mu \int_{0}^{c}\left(\frac{c-x}{p(s)+c}\right)^{1-2 \beta} \frac{\tau(s) p^{\prime}(s) d s}{p(s)+x} .
\end{gathered}
$$

В силу (24)-(28), равенство (23) запишем в виде

$$
\begin{aligned}
& \Gamma(1-2 \beta) D_{x, c}^{2 \beta-1} \Phi_{2}(x)-\mu \int_{0}^{c}\left(\frac{c-x}{p(s)-c}\right)^{1-2 \beta} \frac{\tau(s) p^{\prime}(s) d s}{p(s)-x} \\
& +\mu \int_{0}^{c}\left(\frac{c-x}{p(s)+c}\right)^{1-2 \beta} \frac{\tau(s) p^{\prime}(s) d s}{p(s)+x} \\
& =\frac{\pi(1+\sin (\pi \beta))}{\cos (\pi \beta)} \tau(x)+\int_{0}^{c}\left(\frac{c-x}{c-t}\right)^{1-2 \beta}\left(\frac{1}{t-x}+\frac{1}{t+x}\right) \tau(t) d t .
\end{aligned}
$$

Равенство (29) перепишем в виде

$$
\begin{aligned}
& \tau(x)+\lambda \int_{0}^{c}\left(\frac{c-x}{c-t}\right)^{1-2 \beta}\left(\frac{1}{t-x}+\frac{1}{t+x}\right) \tau(t) d t \\
& =-\lambda \mu \int_{0}^{c}\left(\frac{c-x}{p(s)-c}\right)^{1-2 \beta} \frac{\tau(s) p^{\prime}(s) d s}{p(s)-x} \\
& +\lambda \mu \int_{0}^{c}\left(\frac{c-x}{p(s)+c}\right)^{1-2 \beta} \frac{\tau(s) p^{\prime}(s) d s}{p(s)+x}+\Phi_{3}(x), x \in[0, c],
\end{aligned}
$$


где $\lambda=\frac{\cos (\pi \beta)}{\pi(1+\sin (\pi \beta))}, \Phi_{3}(x)=\lambda \Gamma(1-2 \beta) D_{x, c}^{2 \beta-1} \Phi_{2}(x)$. Равенство (30) запишем в виде

$$
\begin{aligned}
& \tau(x)+\lambda \int_{0}^{c}\left(\frac{c-x}{c-t}\right)^{1-2 \beta}\left(\frac{1}{t-x}+\frac{1}{t+x}\right) \tau(t) d t \\
& =-\lambda \mu \int_{0}^{c}\left(\frac{c-x}{p(s)-c}\right)^{1-2 \beta} \frac{\tau(s) p^{\prime}(s) d s}{p(s)-x}+R[\tau]+\Phi_{3}(x), x \in[0, c],
\end{aligned}
$$

где

$$
R[\tau]=\lambda \mu \int_{0}^{c}\left(\frac{c-x}{p(s)+c}\right)^{1-2 \beta} \frac{\tau(s) p^{\prime}(s) d s}{p(s)+x}
$$

-регулярный оператор. Первый интегральный оператор правой части (31) не является регулярным, так как подынтегральное выражение при $x=c, s=c$ имеет изолированную особенность первого порядка, поэтому это слагаемое в (31) выделено отдельно. Временно считая правую часть уравнения (31) известной функцией, перепишем его в виде

$$
\tau(x)+\lambda \int_{0}^{c}\left(\frac{c-x}{c-t}\right)^{1-2 \beta}\left(\frac{1}{t-x}+\frac{1}{t+x}\right) \tau(t) d t=g_{0}(x), x \in[0, c],
$$

где

$$
g_{0}(x)=-\lambda \mu \int_{0}^{c}\left(\frac{c-x}{p(s)-c}\right)^{1-2 \beta} \frac{\tau(s) p^{\prime}(s) d s}{p(s)-x}+R[\tau]+\Phi_{3}(x) .
$$

Полагая $(c-x)^{2 \beta-1} \tau(x)=\rho(x),(c-x)^{2 \beta-1} g_{0}(x)=g_{1}(x)$, уравнение (32) запишем в виде

$$
\rho(x)+\lambda \int_{0}^{c}\left(\frac{1}{t-x}+\frac{1}{t+x}\right) \rho(t) d t=g_{1}(x), x \in[0, c] .
$$

В (34) произведя замену $t^{2}=s, x^{2}=\xi, \rho(x)=\rho(\sqrt{\xi})=\rho_{1}(\xi), g_{1}(x)=g_{1}(\sqrt{\xi})=g_{2}(\xi)$, получим сингулярное интегральное уравнение

$$
\rho_{1}(\xi)+\lambda \int_{0}^{c^{2}} \frac{\rho_{1}(s) d s}{s-\xi}=g_{2}(\xi), \xi \in\left[0, c^{2}\right] .
$$

Решение уравнения (35) будем искать в классе функций, удовлетворяющих условию Гельдера на $\left(0, c^{2}\right)$ и ограниченных при $\xi=0$, а при $\xi=c^{2}$ могущих обращаться в бесконечность порядка меньше $1-2 \beta$. Единственное решение уравнения (35) в классе $h(0)$ выражается формулой

$$
\begin{aligned}
& \rho_{1}(\xi)=\frac{1+\sin (\beta \pi)}{2} g_{2}(\xi)-\frac{\cos (\beta \pi)}{2 \pi}\left(\frac{\xi}{c^{2}-\xi}\right)^{\frac{1}{4}(1-2 \beta)} \\
& \times \int_{0}^{c^{2}} \frac{g_{2}(t) d t}{\left(\frac{t}{c^{2}-t}\right)^{\frac{1}{4}(1-2 \beta)}(t-\xi)} .
\end{aligned}
$$

Возвращаясь к прежним переменным и функциям из (36), имеем

$$
\begin{aligned}
& \tau(x)=\cos ^{2}(\pi \alpha) g_{0}(x)-\frac{\sin (2 \pi \alpha)}{2 \pi} \\
& \times \int_{0}^{c}\left(\frac{x}{t}\right)^{2 \alpha}\left(\frac{c+t}{c+x}\right)^{\alpha}\left(\frac{c-x}{c-t}\right)^{3 \alpha}\left(\frac{1}{t-x}+\frac{1}{t+x}\right) g_{0}(t) d t,
\end{aligned}
$$


где $\alpha=(1-2 \beta) / 4$. Подставляя (33) в (37), имеем

$$
\begin{aligned}
& \tau(x)=-\lambda \mu \cos ^{2}(\lambda \pi) \int_{0}^{c}\left(\frac{c-x}{p(s)-c}\right)^{4 \alpha} \frac{p^{\prime}(s) \tau(s) d s}{p(s)-x} \\
& +\lambda \mu \frac{\sin (2 \pi \alpha)}{2 \pi} \int_{0}^{c} \tau(s) p^{\prime}(s) d s \int_{0}^{c}\left(\frac{x}{t}\right)^{2 \alpha}\left(\frac{c+t}{c+x}\right)^{\alpha}\left(\frac{c-x}{c-t}\right)^{3 \alpha} \\
& \times\left(\frac{1}{t-x}+\frac{1}{t+x}\right)\left(\frac{c-t}{p(s)-c}\right)^{4 \alpha} \frac{d t}{p(s)-t}+R_{1}[\tau]+\Phi_{4}(x),
\end{aligned}
$$

где

$$
\begin{aligned}
R_{1}[\tau]=\cos ^{2}(\pi \alpha) R[\tau] & -\frac{\sin (2 \pi \alpha)}{2 \pi} \int_{0}^{c}\left(\frac{x}{t}\right)^{2 \alpha}\left(\frac{c+t}{c+x}\right)^{\alpha}\left(\frac{c-x}{c-t}\right)^{3 \alpha} \\
& \times\left(\frac{1}{t-x}+\frac{1}{t+x}\right) R[\tau] d t
\end{aligned}
$$

- регулярный оператор,

$$
\begin{gathered}
\Phi_{4}(x)=\cos ^{2}(\pi \alpha) \Phi_{3}(x)-\frac{\sin (2 \pi \alpha)}{2 \pi} \int_{0}^{c}\left(\frac{x}{t}\right)^{2 \alpha}\left(\frac{c+t}{c+x}\right)^{\alpha}\left(\frac{c-x}{c-t}\right)^{3 \alpha} \\
\times\left(\frac{1}{t-x}+\frac{1}{t+x}\right) \Phi_{3}(t) d t
\end{gathered}
$$

- известная функция. Уравнение (38) запишем в виде

$$
\begin{aligned}
& \tau(x)=-\lambda \mu \cos ^{2}(\lambda \pi) \int_{0}^{c}\left(\frac{c-x}{p(s)-c}\right)^{4 \alpha} \frac{p^{\prime}(s) \tau(s) d s}{p(s)-x} \\
& +\lambda \mu \frac{\sin (2 \pi \alpha)}{2 \pi} \int_{0}^{c} \tau(s) p^{\prime}(s) d s \int_{0}^{c}\left(\frac{x}{t}\right)^{2 \alpha}\left(\frac{c-x}{c-t}\right)^{3 \alpha} \\
& \times\left(\frac{c-t}{p(s)-c}\right)^{4 \alpha}\left(\frac{1}{t-x}+\frac{1}{t+x}\right) \frac{d t}{p(s)-t}+R_{2}[\tau]+\Phi_{4}(x), x \in(0, c),
\end{aligned}
$$

где

$$
\begin{gathered}
R_{2}[\tau]=R_{1}[\tau]+\lambda \mu \frac{\sin (2 \pi \alpha)}{2 \pi} \int_{0}^{c} \tau(s) p^{\prime}(s) d s \\
\times \int_{0}^{c}\left(\frac{x}{t}\right)^{2 \alpha}\left(\frac{c-x}{c-t}\right)^{3 \alpha}\left(\frac{c-t}{p(s)-c}\right)^{4 \alpha}\left[\left(\frac{c+t}{c+x}\right)^{\alpha}-1\right]\left(\frac{1}{t-x}+\frac{1}{t+x}\right) \frac{d t}{p(s)-t}
\end{gathered}
$$

- регулярный оператор. В силу $p(x)=1-k x$, уравнение (39) перепишем в виде

$$
\begin{aligned}
& \tau(x)=\lambda \mu k^{1-4 \alpha} \cos ^{2}(\lambda \pi) \int_{0}^{c}\left(\frac{c-x}{c-s}\right)^{4 \alpha} \frac{\tau(s) d s}{1-k s-x} \\
& -\lambda \mu k^{1-4 \alpha} \frac{\sin (2 \pi \alpha)}{2 \pi} \int_{0}^{c} \tau(s) \frac{d s}{(c-s)^{4 \alpha}} \int_{0}^{c}\left(\frac{x}{t}\right)^{2 \alpha}\left(\frac{c-x}{c-t}\right)^{3 \alpha} \\
& \times(c-t)^{4 \alpha}\left(\frac{1}{t-x}+\frac{1}{t+x}\right) \frac{d t}{1-k s-t}+R_{2}[\tau]+\Phi_{4}(x), x \in(0, c) .
\end{aligned}
$$


Далее, нетрудно убедиться в том, что значение внутреннего интеграла в (40) имеет вид

$$
\begin{aligned}
& A(x, s)=\int_{0}^{c}\left(\frac{x}{t}\right)^{2 \alpha}(c-x)^{3 \alpha}(c-t)^{\alpha}\left(\frac{1}{t-x}+\frac{1}{t+x}\right) \frac{d t}{1-k s-t} \\
& =\frac{(c-x)^{3 \alpha} x^{2 \alpha}}{1-k s-x}\left(\frac{(c-x)^{\alpha}}{x^{2 \alpha}} \pi c t g(\pi \alpha)-\frac{c^{\alpha}}{x^{2 \alpha}} \frac{\Gamma(1-2 \alpha) \Gamma(\alpha)}{\Gamma(1-\alpha)} F\left(1-2 \alpha,-\alpha, 1-\alpha ; \frac{c-x}{c}\right)\right) \\
& +\frac{(c-x)^{3 \alpha} x^{2 \alpha}}{1-k s-x} \\
& \times\left(\frac{c^{1-\alpha}}{1-k s} \frac{\Gamma(1-2 \alpha) \Gamma(\alpha)}{\Gamma(1-\alpha)} F\left(1-2 \alpha, 1,1-\alpha ; \frac{k(c-s)}{1-k s}\right)-\Gamma(\alpha) \Gamma(1-\alpha) \frac{k^{\alpha}(c-s)^{\alpha}}{(1-k s)^{2 \alpha}}\right) \\
& +B_{0}(x, s)
\end{aligned}
$$

где

$$
\begin{gathered}
B_{0}(x, s)=\frac{(c-x)^{3 \alpha} x^{2 \alpha}}{1-k s+x}\left(\frac{c^{1-\alpha}}{x^{2 \alpha}(c+x)^{1-2 \alpha}} \frac{\Gamma(1-2 \alpha) \Gamma(1+\alpha)}{\Gamma(2-\alpha)} F\left(1-\alpha, 1-\alpha, 2-\alpha ; \frac{c}{c+x}\right)\right) \\
+\frac{(c-x)^{3 \alpha} x^{2 \alpha}}{1-k s+x} \\
\times\left(\frac{c^{1-\alpha}}{1-k s} \frac{\Gamma(1-2 \alpha) \Gamma(\alpha)}{\Gamma(1-\alpha)} F\left(1-2 \alpha, 1,1-\alpha ; \frac{k(c-s)}{1-k s}\right)-\Gamma(\alpha) \Gamma(1-\alpha) \frac{k^{\alpha}(c-s)^{\alpha}}{(1-k s)^{2 \alpha}}\right),
\end{gathered}
$$

здесь $B_{0}(x, s)$ - непрерывно-дифференцируемая функция в квадрате $[0, c] \times[0, c]$. Теперь, подставляя (41) в (40), получим

$$
\begin{aligned}
& \tau(x)=\lambda \mu k^{1-3 \alpha} \cos (\pi \alpha) \int_{0}^{c}\left(\frac{c-x}{c-s}\right)^{3 \alpha}\left(\frac{x}{1-k s}\right)^{2 \alpha} \frac{\tau(s) d s}{1-k s-x} \\
& +R_{3}[\tau]+\Phi_{4}(x), x \in(0, c)
\end{aligned}
$$

где

$$
\begin{gathered}
R_{3}[\tau]=R_{2}[\tau]+\lambda \mu k^{1-4 \alpha} \frac{\sin (2 \pi \alpha)}{2 \pi} \frac{\Gamma(1-2 \alpha) \Gamma(\alpha)}{\Gamma(1-\alpha)} \int_{0}^{c} \frac{\tau(s)(c-x)^{3 \alpha} c^{\alpha}}{(c-s)^{4 \alpha}(1-k s-x)} \\
\times\left(c^{\alpha} F\left(1-2 \alpha,-\alpha, 1-\alpha ; \frac{c-x}{c}\right)-x^{2 \alpha} \frac{c^{1-\alpha}}{1-k s} F\left(1-2 \alpha, 1,1-\alpha ; \frac{k(c-x)}{1-k s}\right) d s\right)- \\
-\lambda \mu k^{1-4 \alpha} \frac{\sin (2 \pi \alpha)}{2 \pi} \int_{0}^{c} \frac{B_{0}(x, s) \tau(s) d s}{(c-s)^{4 \alpha}}
\end{gathered}
$$

-регулярный оператор. Равенство (42) запишем в виде

$$
\tau(x)=\lambda \mu k^{1-3 \alpha} \cos (\pi \alpha) \int_{0}^{c}\left(\frac{c-x}{c-s}\right)^{3 \alpha} \frac{\tau(s) d s}{1-k s-x}+R_{4}[\tau]+\Phi_{4}(x), x \in(0, c),
$$

где

$$
R_{4}[\tau]=R_{3}[\tau]+\lambda \mu k^{1-3 \alpha} \cos (\pi \alpha) \int_{0}^{c}\left(\frac{c-x}{c-s}\right)^{3 \alpha} \frac{\tau(s)}{1-k s-x}\left[\left(\frac{x}{1-k s}\right)^{2 \alpha}-1\right] d s
$$


-регулярный оператор. Уравнение (43) перепишем в виде

$$
\tau(x)=\lambda \mu k^{1-3 \alpha} \cos (\pi \alpha) \int_{0}^{c}\left(\frac{c-x}{c-s}\right)^{3 \alpha} \frac{\tau(s) d s}{(c-s)\left[k+\frac{c-x}{c-s}\right]}+R_{4}[\tau]+\Phi_{4}(x), x \in(0, c) .
$$

После замены переменных $x=c-c e^{-\xi}, s=c-c e^{-t}$ и введения обозначения

$$
\begin{aligned}
\rho(\xi)= & \tau\left(c-c e^{-\xi}\right) e^{\left(3 \alpha-\frac{1}{2}\right) \xi} \text { уравнение (44) принимает вид } \\
& \rho(\xi)=\lambda \mu k^{1-3 \alpha} \cos (\pi \alpha) \int_{0}^{\infty} \frac{\tau(t) d t}{k e^{\frac{\xi-t}{2}}+e^{-\frac{\xi-t}{2}}}+R_{5}[\tau]+\Phi_{5}(\xi), \xi \in(0, \infty),
\end{aligned}
$$

где $R_{5}[\tau]=R_{4}[\tau] e^{\left(3 \alpha-\frac{1}{2}\right) \xi}$ - регулярный оператор, $\Phi_{5}(\xi)=\Phi_{4}\left(c-c e^{-\xi}\right) e^{\left(3 \alpha-\frac{1}{2}\right) \xi}$. Заметим, что в силу условия $\beta_{0}>\frac{1-m}{3}$ имеет место неравенство $6 \alpha-1<0$.

Введем обозначение

$$
N(\xi)=\frac{\lambda \mu k^{1-3 \alpha} \cos (\alpha \pi)}{k e^{\frac{\xi}{2}}+e^{-\frac{\xi}{2}}} .
$$

Тогда уравнение (45) запишем в виде

$$
\rho(\xi)=\int_{0}^{\infty} N(\xi-t) \rho(t) d t+R_{5}[\tau]+\Phi_{5}(\xi), \xi \in(0, \infty) .
$$

Уравнение (46) является интегральным уравнением Винера-Хопфа [9] и с помощью преобразования Фурье оно приводится к краевой задаче Римана, т.е. решается в квадратурах. Функции $N(\xi), \Phi_{5}(\xi)$ имеют показательный порядок убывания на бесконечности, причём $N(\xi) \in C(0, \infty), \Phi_{5}(\xi) \in H_{\alpha_{1}}(0, \infty)$. Следовательно, $N(\xi)$, $\Phi_{5}(\xi) \in L_{2} \cap H_{\alpha_{1}}$. Теоремы Фредгольма для интегральных уравнений типа свертки справедливы лишь в одном частном случае, когда индекс этих уравнений равен нулю. Индексом уравнения (46) будет индекс выражения $1-N^{\wedge}(\xi)$ с обратным знаком, где

$$
N^{\wedge}(\xi)=\int_{-\infty}^{\infty} e^{i \xi t} N(t) d t=\lambda \mu k^{1-3 \alpha} \cos (\alpha \pi) \int_{-\infty}^{\infty} \frac{e^{i \xi t} d t}{k e^{\frac{t}{2}}+e^{-\frac{t}{2}}}
$$

Вычислив интеграл Фурье, с помощью теории вычетов [6] найдем

$$
\int_{-\infty}^{\infty} \frac{e^{i \xi t} d t}{k e^{\frac{t}{2}}+e^{-\frac{t}{2}}}=\frac{\pi e^{-i \xi \ln k}}{\sqrt{k} \operatorname{ch}(\pi \xi)}
$$

Подставляя (48) в (47), в силу $\lambda=\frac{\cos (\beta \pi)}{\pi(1+\sin (\beta \pi))}, \alpha=(1-2 \beta) / 4$, имеем

$$
N^{\wedge}(\xi)=\mu k^{\frac{1}{2}-3 \alpha} \sin (\alpha \pi) \frac{e^{-i \xi \operatorname{lnk}}}{\operatorname{ch}(\pi \xi)}
$$

Поскольку $\mu k^{\frac{1}{2}-3 \alpha} \sin (\pi \alpha)<1$ и так как

$$
\begin{gathered}
\operatorname{Re}\left(N^{\wedge}(\xi)\right)=\operatorname{Re}\left(\mu k^{\frac{1}{2}-3 \alpha} \sin (\alpha \pi) \frac{e^{-i \xi \ln k}}{\operatorname{ch}(\pi \xi)}\right) \\
=\mu k^{\frac{1}{2}-3 \alpha} \sin (\pi \alpha) \frac{\cos (\xi \ln k)}{\operatorname{ch}(\pi \xi)}<\mu k^{\frac{1}{2}-3 \alpha} \sin (\pi \alpha)<1,
\end{gathered}
$$


то $\operatorname{Re}\left(1-N^{\wedge}(\xi)\right)>0$. Следовательно, индекс уравнения (46) $\chi=-\operatorname{Jnd}\left(1-N^{\wedge}(\xi)=0\right.$, т.е. изменение аргумента выражения $\left.1-N^{\wedge}(\xi)\right)$ на действительной оси,выраженное в полных оборотах, равно нулю [9]. Следовательно, уравнение (46) однозначно редуцируется к интегральному уравнению Фредгольма второго рода, однозначная разрешимость которого следует из единственности решения задачи $B$.

Конкурирующие интересы. Авторы заявляют, что конфликтов интересов в отношении авторства и публикации нет.

Авторский вклад и ответственность. Авторы участвовали в написании статьи и полностью несут ответственность за представление окончательной версии статьи в печать.

\section{Список литературы/References}

[1] Смирнов М. М., Уравнения смешанного типа, Высшая школа, М., 1985, 304 с. [Smirnov M.M., Mixed type equations, Higher school, Moscow, 1985, 304 pp.]

[2] Репин О. А., Кумыкова С. К., “Об одной краевой задаче со смещением для уравнения смешанного типа в неограниченной области”, Дифферен. уравнения, 48:8 (2012), 11401149. [Repin O.A., Kumykova S.K., "On a boundary value problem with shift for an equation of mixed type in an unbounded domain", Differ. Equations, 48:8 (2012), 1127$1136]$.

[3] Рузиев M. Х., “Краевая задача для уравнения смешанного типа с сингулярным коэффициентом в области, эллиптическая часть которой - полуполоса”, $B e c m$. Сам.гос.тех.ун-та. Сер.физ.-мат.науки, 18:1 (2009), 33-40. [Ruziev M. Kh., "Boundary value problem for the equation of mixed type with singular coefficient in the domain where the elliptic part is a half-band", Journal of Samara State Technical University, Ser. Physical and Mathematical Sciences, 18:1(18) (2009), 33-40].

[4] Gellerstedt S., "Quelques problemes mixtes pour 1'equation $y^{m} z_{x x}+z_{y y}=0$ ", Ark. Math. Astron. Fys., 26A:3 (1938), 1-32.

[5] Рузиев М. Х., “О нелокальной задаче для уравнения смешанного типа с сингулярным коэффициентом в неограниченной области”, Нзв.вузов. Матем., 2010, № 11, 41-49. [Ruziev M. Kh., "A nonlocal problem for a mixed-type equation with a singular coefficient in an unbounded domain”, Russian Mathematics, 2010, № 54, 36-43].

[6] Мирсабуров M., Рузиев M. Х., “Об одной краевой задаче для одного класса уравнений смешанного типа в неограниченной области”, Дифферен. уравнения, 47:1 (2011), 112119. [Mirsaburov M, Ruziev M. Kh., "A boundary value problem for a class of mixed type equations in an unbounded domain”, Differ. Equations, 47:1 (2011), 111-118].

[7] Лернер М. Е., Пулькин С. П., “О единственности решений задач с условиями Франкля и Трикоми для общего уравнения Лаврентьева-Бицадзе”, Дифферен. уравнения, 11:9 (1966), 1255-1263. [Lerner M.E., Pul'kin S. P., "On uniquness of solutions of problems satisfying the Frankl and Tricomi conditions for the general Lavrent'ev-Bitsadze equation", Differ. Equations, 2:9 (1966), 1255-1263].

[8] Бицадзе А. В., Некоторые классы уравнений в частных производных, Наука, М., 1981, 448 c. [Bitsadze A. V., Some Classes of Partial Differential Equations, Science, Moscow, 1981, 448 pp.]

[9] Гахов Ф. D., Черский Ю. И., Уравнения типа свертки, Наука, М., 1978, 295 с. [Gakhov F. D., Chersky Yu. I., Convolution type equations, Science, Moscow, 1978, 295 pp.] 


\title{
A boundary value problem for mixed type equation with singular coefficient
}

\author{
M. Kh. Ruziev' ${ }^{1}$ F.S. Aktamov ${ }^{2}$ \\ 1 Institute of Mathematics Uzbekistan Academy of Sciences, 100174, Tashkent, \\ University st., 4b, Uzbekistan \\ 2 Chirchik State Pedagogical Institute, Amir Temur avenue, 104, Uzbekistan \\ E-mail: mruziev@mail.ru \\ In this paper we study a boundary value problem for a mixed type equation in a domain \\ whose elliptic part is the first quadrant of the plane and the hyperbolic part is the char- \\ acteristic triangle. With the help of the method of integral equations and the principle of \\ extremum we prove the unique solvability of the considered problem.
}

Key words: principle of extremum, unique solvability, solvability, singular coefficient, index of equation, integral equations.

DOI: $10.26117 / 2079-6641-2021-35-2-27-39$

Original article submitted: 24.04 .2021

Revision submitted: 04.06.2021

For citation. Ruziev M. Kh., Aktamov F.S. A boundary value problem for mixed type equation with singular coefficient. Vestnik KRAUNC. Fiz.-mat. nauki. 2021,35: 2,27-39. DOI: 10.26117/2079-6641-2021-35-2-27-39

Competing interests. The authors declare that there are no conflicts of interest regarding authorship and publication.

Contribution and Responsibility. All authors contributed to this article. Authors are solely responsible for providing the final version of the article in print. The final version of the manuscript was approved by all authors.

The content is published under the terms of the Creative Commons Attribution 4.0 International License (https://creativecommons.org/licenses/by/4.0/deed.ru)

(C) Ruziev M. Kh., Aktamov F. S., 2021

Funding. The study was carried out without financial support from foundations. 